\title{
Electronic structure of boron doped diamond: An x-ray spectroscopic study
}

\author{
P.-A. Glans, ${ }^{1,2}$ T. Learmonth, ${ }^{1}$ K. E. Smith, ${ }^{1}$ S. Ferro, ${ }^{3}$ A. De Battisti, ${ }^{3}$ M. Mattesini, ${ }^{4,5}$ \\ R. Ahuja, ${ }^{6}$ and J.-H. Guo ${ }^{2, a)}$ \\ ${ }^{1}$ Department of Physics, Boston University, 590 Commonwealth Ave., Boston, Massachusetts 02215, USA \\ ${ }^{2}$ Advanced Light Source, Lawrence Berkeley National Laboratory, I Cyclotron Rd, Berkeley, \\ California 94720, USA \\ ${ }^{3}$ Department of Chemistry, University of Ferrara, via L. Borsari 46, I-44100 Ferrara, Italy \\ ${ }^{4}$ Departamento de Física de la Tierra, Astronomía y Astrofísica I, Universidad Complutense de Madrid, \\ E-28040 Madrid, Spain \\ ${ }^{5}$ Instituto de Geociencias (UCM-CSIC), Facultad de Ciencias Físicas, Plaza de Ciencias 1, 28040 Madrid, \\ Spain \\ ${ }^{6}$ Department of Physics and Astronomy, Uppsala University, Box 516, SE-751 20 Uppsala, Sweden
}

(Received 15 June 2012; accepted 9 April 2013; published online 23 April 2013)

\begin{abstract}
The valence and conduction band electronic structure of boron-doped diamond has been measured using soft $\mathrm{x}$-ray emission and absorption spectroscopy. The experimental results reveal $p$-type doping in the diamond film through the appearance of states in the band-gap. Structure distortion was observed around the doping center, while the long range order of the diamond structure remains. A chemically shifted C $1 s$ level explains why one of the absorption features seems to appear below the valence band maximum. An excitonic feature was observed in the boron-doped diamond, similar to that observed in pure diamond, indicating that the exciton binding energy remains the same upon B-doping. (C) 2013 AIP Publishing LLC [http://dx.doi.org/10.1063/1.4802814]
\end{abstract}

Diamond is a wide band-gap material $(5.47 \mathrm{eV})$ that is being explored for possible optoelectronic applications. Cathodoluminescence and photoluminescence measurements show several emission features in the ultraviolet (UV), at considerably higher energies than those observed from gallium nitride. This makes diamond very interesting as a semiconductor because it allows for the possibility of UV light-emitting devices. ${ }^{1}$ Device applications require thin films of diamond, grown in a controlled environment. Processes for the hot filament chemical vapor deposition (HFCVD) of diamond thin films have their origin in Eversole's patents, in which the thermal decomposition of carbon-containing gases was used to grow diamond films on natural diamond crystals heated from approximately $600-1600{ }^{\circ} \mathrm{C}{ }^{2}$ When heavily doped diamond films are required, the dopant atoms are introduced by supplying appropriate chemical species to the process gas. In general, boron is inserted into the diamond lattice to realize $p$-type doping. The resulting films are hard, chemically inert, wear resistant, and semiconducting. In this complex frame, comprehensive measurements of the electronic structure of the films are very important.

We report a measurement of the valence and conduction band electronic structure of boron doped diamond (BDD) films using high-resolution soft $\mathrm{x}$-ray emission (XES) and absorption spectroscopy (XAS). This combination of spectroscopies has been shown earlier to provide important information about the electronic structure both of pure diamond and of highly ordered pyrolytic graphite (HOPG). ${ }^{3,4}$ Because a core level is involved, both XES and XAS are element specific. XES reflects the occupied partial density of states (PDOS), while XAS reflects the unoccupied PDOS. Applied together, these techniques deliver valuable information

\footnotetext{
${ }^{\text {a) }}$ Author to whom correspondence should be addressed. Electronic mail: jguo@lbl.gov
}

about the nature and evolution of the band-gap in wide gap materials, as has been shown for $\mathrm{Al}_{x} \mathrm{Ga}_{1-x} \mathrm{~N},{ }^{5} \operatorname{In}_{x} \mathrm{Ga}_{1-x} \mathrm{~N},{ }^{6}$ $\mathrm{SiO}_{x} \mathrm{~N}_{y},{ }^{7}$ and $\mathrm{SiC}^{8}$ XES and XAS have been applied to BDD in an earlier study, but only films with a low level of boron doping were studied. ${ }^{9}$

A measurement of the exciton binding energy in BDD is also reported. Pure diamond possesses an exciton, which has previously been studied using XAS, ${ }^{4,10-12}$ which in turn gives access to the binding energy of the unrelaxed exciton. Including phonon losses, which are not accessible by XAS, an accurate determination of the binding energy of the relaxed exciton can be made. Recording x-ray emission spectra with excitation energies resonant with a core level, additional features appear in the spectra due to valence excitations rather than simply the PDOS fluorescence. Operated in this mode, the spectroscopy is known as resonant inelastic X-ray scattering (RIXS). RIXS has been used by Ma et al. to determine that the binding energy of the relaxed exciton in pure diamond is $1.5 \mathrm{eV} .{ }^{4}$ Our RIXS measurements show that the binding energy of the exciton in BDD remains the same as that in pure diamond.

The experiments were performed at undulator beam-line 7.0.1 at the Advanced Light Source, Lawrence Berkeley National Laboratory. The beam-line is equipped with a spherical grating monochromator. ${ }^{13}$ The XES spectra were collected using a Nordgren type spectrometer. ${ }^{14}$ The XAS spectra were recorded in fluorescence yield (FY) mode by using a channeltron. The incident photon energy resolution was set to $50 \mathrm{meV}$ for the $\mathrm{C} K$-edge XAS measurements and to $100 \mathrm{meV}$ for the XES measurements. The resolution of the $\mathrm{X}$-ray emission spectrometer was set to $300 \mathrm{meV}$ for the $\mathrm{C} K$ edge. The incidence angle (angle between the incoming photon beam and the sample surface) was $25^{\circ}$. The energy scale of the C $1 \mathrm{~s}$ absorption spectra were calibrated to the $\pi^{*}$ peak at $285.5 \mathrm{eV}$ in XAS of HOPG. ${ }^{15}$ The emission spectra were 
calibrated by aligning the resonant elastic peak in the spectra to the correct energy in the XAS. Three types of BDD samples were investigated: (i) as-grown samples synthesized by the HFCVD technique in the laboratories of Centre Suisse d'Electronique et de Microtechnique SA (CSEM) (Neuchâtel, Switzerland), (ii) annealed samples, heated ex-situ at $400^{\circ} \mathrm{C}$ for $30 \mathrm{~min}$, and (iii) samples that had been fluorinated by exposure to a flux of $\mathrm{CF}_{4}$ plasma. All films had a thickness of approximately $1 \mu \mathrm{m}$ and consisted of micro-crystals with grain sizes in the range of $0.1-0.5 \mu \mathrm{m} .{ }^{16}$ The as-grown sample had a doping level of $3500 \mathrm{ppm}$ as determined by resistivity measurements and comparing to previous measurements. See Refs. 16 and 17 for detailed information about the samples.

Figure 1 presents $\mathrm{C} K$-edge XES and XAS spectra from both an as-grown BDD film and a sample of HOPG. These spectra primarily reflect the C $2 p$ PDOS of the valence and conduction bands, respectively. The spectral features of note in the BDD XAS spectra are labeled $a-e$ in Fig. 1. Of particular importance is the observation that doping of the diamond film induces absorption features in the fundamental bandgap $(a, b$, and $c)$. These localized states are ascribed to the presence of the electron-deficient B atoms in the film. Features $a$ and $b$ are at $282.5 \mathrm{eV}$ and $284.0 \mathrm{eV}$ respectively, also observed by other groups, ${ }^{9,18}$ and lie below the valence band maximum (VBM) as determined from extrapolation of the leading edge of the XES spectrum. Feature $c$ at $285.2 \mathrm{eV}$ is very close in energy to the $\pi^{*}$ peak of graphite $(285.5 \mathrm{eV})$. In a perfect diamond structure, there are only $s p^{3}(\sigma)$ bonds and no $s p^{2}(\pi)$ bonds, but if the boron doping introduces structural changes in the lattice, such as lattice distortions, it is likely that $\pi$ bonds appear. The $\pi^{*}$ feature has also been observed in films of nano-diamond, ${ }^{19}$ in diamond films with grain sizes up to $5 \mu \mathrm{m},{ }^{10}$ and in bulk diamond with defects. ${ }^{20}$ Thus, we identify peak $c$ as a graphite-like $\pi^{*}$ feature due to lattice defects. The sharp feature $d$ in Fig. 1 at $288.9 \mathrm{eV}$ is the diamond exciton. ${ }^{12}$ Feature $e$ is at the energy where the second absolute band-gap would be in diamond. ${ }^{3,21}$

There is an interesting difference in the valence band PDOS for BDD compared to that of pure diamond. A small shoulder is observed just at the edge of the VBM in the XES spectrum of BDD, indicating an increased DOS at the VBM compared to diamond, which could be responsible for the increased conductivity in BDD. Nakamura et al. performed a

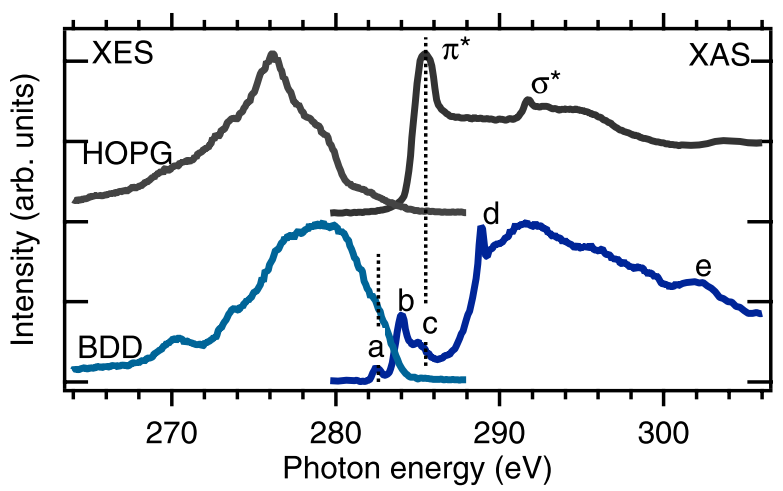

FIG. 1. Experimental spectra of HOPG and the as-grown BDD. The excitation energy for the emission spectra was $320 \mathrm{eV}$. The $\pi^{*}$ feature in the graphite spectrum has been used for calibration. Three features, $a, b$ and $c$, are created in the band-gap of boron doped diamond. discrete variational $\mathrm{X} \alpha(\mathrm{DVX} \alpha)$ calculation of a $\mathrm{C}_{174} \mathrm{BH}_{16}$ cluster. $^{9}$ In the case of substitutional boron, the calculation shows that B $2 p$ has a high PDOS at the VBM-close to the energy where the shoulder is in the XES spectrum in Fig. 1. This feature is an indication of strong hybridization between the $\mathrm{B} 2 p$ and $\mathrm{C} 2 p$ orbitals. ${ }^{9}$

Figure 2 shows XAS of the $\mathrm{C} K$ edge recorded in FY mode. The spectrum marked "As grown" is the same as in Fig. 1; the spectrum marked "Annealed" is from an annealed sample and the spectrum marked "Fluorinated" is from a sample submitted to a flux of $\mathrm{CF}_{4}$ plasma. The three features in the band-gap persist for the annealed sample, but their intensities change. Both features $a$ and $b$ decrease in intensity relative to $c$. The exciton $(d)$ is less pronounced. In the spectrum from the fluorinated sample, both features $a$ and $b$ have basically disappeared. The diamond exciton is again more pronounced and the other high energy features of the XAS are very similar to those found in pure diamond. ${ }^{3,20,21}$ Most notable is the second fundamental band-gap at an absorption energy of $\sim 302 \mathrm{eV}$.

The inset in Fig. 2 shows the XAS of the B $K$ edge recorded in FY mode. The B $1 s$ peak is clearly visible at $\sim 192 \mathrm{eV}$ in the spectra of the as-grown and the annealed samples. In the spectrum from the fluorinated sample, however, the feature is gone. This indicates that fluorination removes the boron to below the detection level from the BDD. The larger probing depth of FY means that the fluorination is not confined only to the surface. Measurements at the F $K$ edge (not shown) exhibits a barely discernible feature in the more surface sensitive Total Electron Yield mode, but no significant $\mathrm{F} K$ signal was observed in the bulk sensitive FY mode.

The second fundamental band-gap in pure diamond is only visible in the spectra from the fluorinated sample. Therefore, feature $e$ in Fig. 1 may also be related to the boron doping. The appearance of feature $e$ is most likely structural due to its relatively high energy.

Features $a$ and $b$ have been shown to be related to the presence of boron, since, as shown in Fig. 2, these two absorption features are missing from the spectrum from the fluorinated sample, and the boron is removed from the same sample, as shown in the inset of Fig. 2. It is possible that

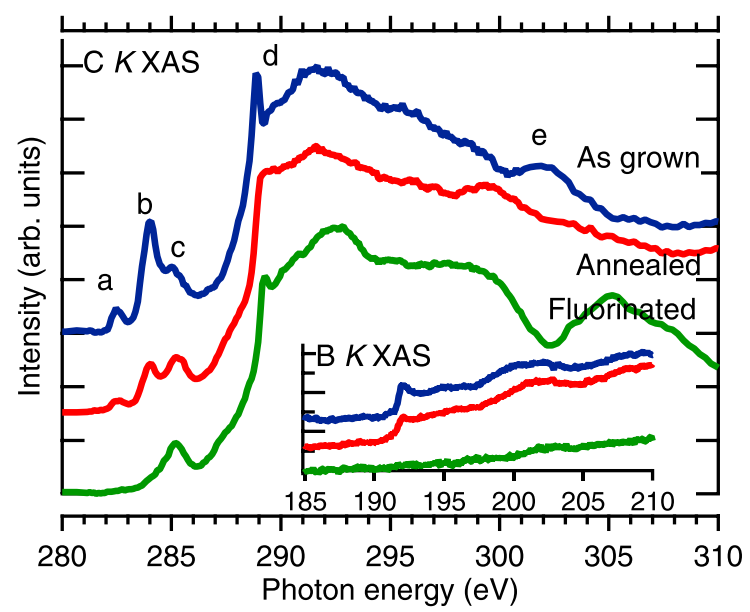

FIG. 2. Experimental XAS spectra of the $\mathrm{C} K$ edge, and of the B $K$ edge (inset). Both recorded in Fluorescence Yield mode. 
these states exist throughout the diamond crystal, but this makes it difficult to explain the low energy of feature $a$. Since both XAS and RIXS are local probes, it is possible that we are picking up signals from carbon atoms in the immediate vicinity of the boron dopants and that their $\mathrm{C} 1 \mathrm{~s}$ levels exhibit a (positive) chemical shift. If the core level shift is large enough, this could make a state close to the VBM appear below the VBM. The $\mathrm{C} 1 s$ levels of the carbon atoms that are nearest neighbors (NNs) to the boron dopants will display a chemical shift towards lower binding energy, ${ }^{22}$ and since XAS measures the energy difference between the core level and the unoccupied state, a chemical shift of a core level can make an unoccupied state measured by XAS appear below the VBM as determined by XES. This makes sense if the state in question exists locally on the atoms that simultaneously exhibit a chemical core level shift. This indicate not only that feature $a$ is related to the presence of boron in the diamond but also that feature $a$ is a state that is localized to the carbon atoms in close proximity to the boron atoms and thus a direct result of the hole doping.

Feature $b$ can be either (i) an excitation from the unshifted core level into the same empty state that gives rise to feature $a$, or (ii) an excitation from the same shifted core level as above into a different empty state in the band-gap. Since XAS and RIXS are local probes, the requirement for case (i) to happen is that the empty state giving rise to feature $a$ have to exist both on carbon atoms that exhibit a chemically shifted C $1 s$ core level and those that do not. The requirement for scenario (ii) to happen is that the empty state created in the band-gap by the boron doping is localized to the carbon atoms closest to the borons, and also that the introduction of boron induces $s p^{2}$ bonds on these carbons. Feature $b$ would then be of the same character as feature $c$, i.e., feature $b$ is due to $s p^{2}$ bonds on the carbons being NNs to the boron atoms, but since these carbons also exhibit a chemically shifted core level, the feature in the spectrum will be recorded at a lower energy than feature $c$.

The seemingly high intensity of features $a$ and $b$, given that the $\mathrm{B}$ doping level is $3500 \mathrm{ppm}$, can be explained by the probing depth being much larger for the B induced states in the gap compared to the bulk states.

Tuning the excitation energy to correspond to feature $a$ in the absorption spectrum gives the emission spectra labeled $a$ in Fig. 3(a). In this case, the only core hole we are creating is in a chemically shifted C $1 s$ level and we are hence only probing the electronic structure at the carbon atoms where this shift exists. The emission spectra from the as-grown and the annealed samples are very similar, while the emission from the fluorinated sample has a much lower intensity. The emission spectra obtained by tuning the excitation energy to feature $b$ are very similar to the emission spectra from feature $a$ below $276.5 \mathrm{eV}$. The emission spectra from both features are also similar to the above threshold emission spectra below this energy (Fig. 3(b)). We can assume that emission at lower energies is dominated by fluorescence from the VB, while emission at higher energies is more likely to contain scattering features. This means that the electronic structure at the sites probed in the $a$ and $b$ cases are very similar. The emission spectra obtained by tuning the excitation energy to feature $c$, however, have a very different character compared

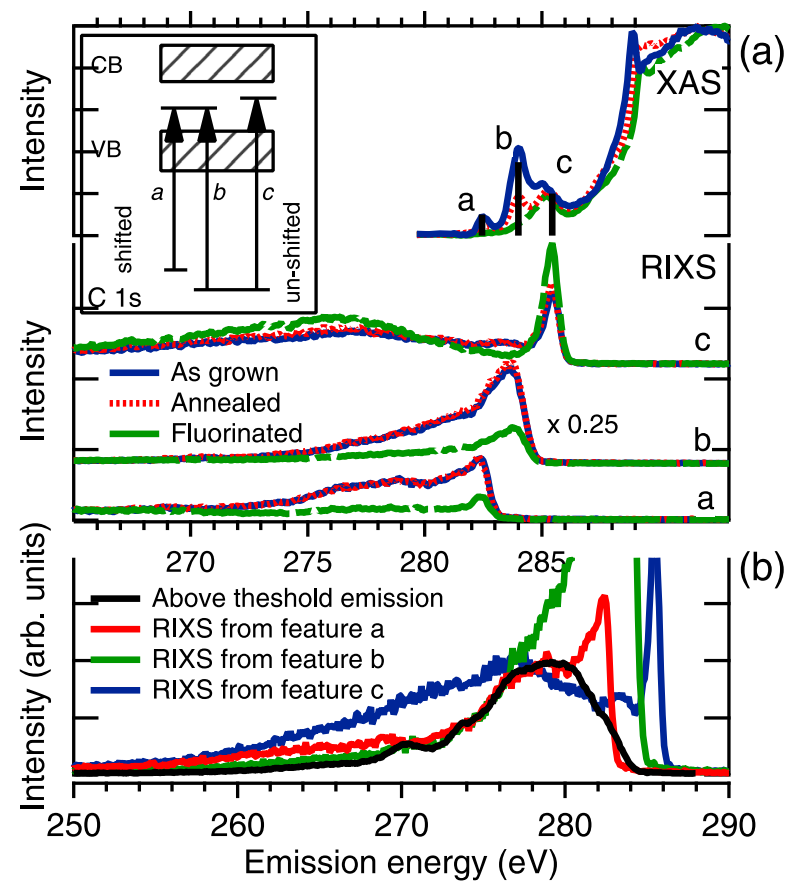

FIG. 3. (a) RIXS measurements of the three states in the band-gap. The RIXS spectra have been normalized to the acquisition time and incoming photon flux. Solid lines (blue) are from the as-grown sample, dotted lines (red) are from the annealed, and the dashed-dotted lines (green) are from the fluorinated sample. (b) A comparison between the RIXS measurements and the above-threshold emission spectrum from the as-grown sample. The RIXS spectra have been scaled to make the intensities of their respective fluorescence parts comparable to the intensity of the above-threshold emission spectrum.

to the other two cases. Hence the electronic structure at the site(s) probed by tuning the excitation energy to feature $c$ is different from the site(s) probed in the feature $a$ and $b$ cases. The states in the band-gap are fairly close in energy to each other, so if we assume that the electronic structure of the VB does not differ substantially between the intermediate states reached in the three cases described above, this means that we must probe a different carbon site for case $c$ compared to the $a$ and $b$ cases. We can assume that the dopant state induced by the presence of B persists further away from the $\mathrm{B}$ than just the NN C atoms. If we also assume that the lattice distortions due to the $\mathrm{B}$ atom are small, then the similar electronic structures in cases $a$ and $b$ would be from $\mathrm{C}$ atoms in the vicinity of a $\mathrm{B}$ atom. The only difference being that only the NN $\mathrm{C}$ atoms to $\mathrm{B}$ are probed in case $a$. When the excitation energy is increased to the $\pi^{*}$ feature, a different $\mathrm{C}$ site is probed. This feature would then be due to vacancies or other lattice distortions, larger than what the presence of the $\mathrm{B}$ atoms induce, in the BDD film. Hence, we conclude that scenario (i) is the most probable. Feature $b$ is likely to be the same state giving rise to feature $a$, except localized on $\mathrm{C}$ atoms slightly further away from the $\mathrm{B}$ atom and not being subject to the same chemical core level shift, see the inset in Fig. 3(a). The energy difference between features $a$ and $b$ would then be a measure of the core level shift for the $\mathrm{C} 1 \mathrm{~s}$ in BDD. We estimate this value to be $1.5 \mathrm{eV}$.

The difference between the diamond exciton feature and the conduction band minimum (CBM) has been quoted at $0.19 \mathrm{eV}$ for a naturally conductive type IIb diamond. ${ }^{12,23}$ 
The binding energy of the relaxed exciton, however, is larger because one has to include the phonon contribution. ${ }^{4,11}$ RIXS measurements (not shown) with the excitation energy tuned to the exciton peak of the as-grown BDD yield very similar features, and in particular the phonon side band, as previously observed in both diamond and graphite. ${ }^{4}$ The exciton visible in the XAS spectrum is evidence of longrange order in the film since the exciton disappears in, for example, nanodiamond ${ }^{24}$ and in diamond with defects. ${ }^{20} \mathrm{Ma}$ et al. explains the phonon-side band as the result of deexcitation of the exciton, so its presence in the RIXS spectra of BDD is further evidence of long-range order in the film. The phonon side-band in BDD is of about the same width as in pure diamond. Since this is the largest contributor to the exciton binding energy, we can conclude that the binding energy of the relaxed exciton in BDD has about the same value as found for diamond $(1.5 \mathrm{eV})$.

In conclusion, we have shown that doping diamond with boron creates states in the fundamental band-gap. Three discrete features are observed (Fig. 1). Features $a$ and $b$ are related to the boron doping, appearing as a direct result of the hole doping. Feature $a$ is indicative of a transition from a chemically shifted core hole to a dopant state in the bandgap of diamond, while feature $b$ appears as a result of the transitions being from un-shifted core holes to the same dopant state. Feature $c$ is identified as being due to a $\pi^{*}$ state. The observation of a $\pi^{*}$ feature and the absence of a second band-gap are interpreted as evidence for a structural change induced by the B atoms in the diamond lattice. Fluorination of the boron doped diamond film removes boron as evidenced by both the features $a$ and $b$ disappears in the $\mathrm{C} K$ $\mathrm{XAS}$, and that the B signal disappears in the B $K$-edge XAS. RIXS measurements of the exciton in BDD show similar features as that in pure diamond, implying a similar exciton binding energy. While some features in the band-gap of BDD is indicative of structural changes induced by the boron doping, the observed phonon side-band is evidence of that the long range order is not destroyed.

The Boston University program is supported by the NSF under DMR 0311792, by the U.S. ARO under PH-45178, and by the U.S. AFOSR. The work at ALS was supported by the U.S. Department of Energy under Contract No. DEAC02-05CH11231.

${ }^{1}$ S. Koizumi, K. Watanabe, M. Hasegawa, and H. Kanada, Science 292, 1899 (2001).

${ }^{2}$ W. G. Eversole, U.S. patent 3030187 (1962); U.S. patent 3030188 (1962).

${ }^{3}$ Y. Ma, N. Wassdahl, P. Skytt, J. Guo, J. Nordgren, P. Johnson, J. E. Rubensson, T. Boske, W. Eberhardt, and S. Kevan, Phys. Rev. Lett. 69, 2598 (1992).

${ }^{4}$ Y. Ma, P. Skytt, N. Wassdahl, P. Glans, J. Guo, and J. Nordgren, Phys. Rev. Lett. 71, 3725 (1993).

${ }^{5}$ L.-C. Duda, C. Stagarescu, J. Downes, K. Smith, D. Korakakis, T. Moustakas, J. Guo, and J. Nordgren, Phys. Rev. B 58, 1928 (1998).

${ }^{6}$ P. Ryan, C. McGuinness, J. Downes, K. Smith, D. Doppalapudi, and T. Moustakas, Phys. Rev. B 65, 205201 (2002).

${ }^{7}$ C. McGuinness, G. Hughes, J. Roche, D. Fu, J. Downes, and K. Smith, J. Appl. Phys. 94, 3919 (2003).

${ }^{8}$ J. Luning, S. Eisebitt, J. Rubensson, C. Ellmers, and W. Eberhardt, Phys. Rev. B 59, 10573 (1999).

${ }^{9}$ J. Nakamura, E. Kabasawa, N. Yamada, Y. Einaga, D. Saito, H. Isshiki, S. Yugo, and R. Perera, Phys. Rev. B 70, 245111 (2004).

${ }^{10}$ Y. Chang, H. Hsieh, W. Pong, M.-H. Tsai, F. Chien, P. Tseng, L. Chen, T. Wang, K. Chen, D. Bhusari, J. Yang, and S. Lin, Phys. Rev. Lett. 82, 5377 (1999).

${ }^{11}$ J. Nithianandam, Phys. Rev. Lett. 69, 3108 (1992).

${ }^{12}$ J. Morar, F. Himpsel, G. Hollinger, G. Hughes, and J. Jordan, Phys. Rev. Lett. 54, 1960 (1985).

${ }^{13}$ T. Warwick, P. Heimann, D. Mossessian, W. McKinney, and H. Padmore, Rev. Sci. Instrum. 66, 2037 (1995).

${ }^{14}$ J. Nordgren, G. Bray, S. Cramm, R. Nyholm, J. Rubensson, and N. Wassdahl, Rev. Sci. Instrum. 60, 1690 (1989).

${ }^{15}$ L. J. Terminello, D. K. Shuh, F. J. Himpsel, D. A. Lapiano-Smith, J. Stöhr, D. S. Bethune, and G. Meijer, Chem. Phys. Lett. 182, 491 (1991); J. Guo and J. Nordgren, J. Electron Spectrosc. Relat. Phenom. 110, 105 (2000).

${ }^{16}$ S. Ferro, J. Mater. Chem. 12, 2843 (2002).

${ }^{17}$ S. Ferro and A. De Battisti, J. Phys. Chem. B 107, 7567 (2003).

${ }^{18}$ I. Zegkinoglou, P. L. Cook, P. S. Johnson, W. Yang, J. Guo, D. Pickup, R. González-Moreno, C. Rogero, R. E. Ruther, M. L. Rigsby, J. E. Ortega, R. J. Hamers, and F. J. Himpsel, J. Phys. Chem. C 116, 13877 (2012).

${ }^{19}$ L. Chen, T. Wang, J. Yang, K. Chen, D. Bhusari, Y. Chang, H. Hseih, and W. Pong, Diamond Relat. Mater. 9, 877 (2000); Y. Tang, X. Zhou, Y. Hu, C. Lee, S. Lee, and T. Sham, Chem. Phys. Lett. 372, 320 (2003).

${ }^{20}$ A. Laikhtman, I. Gouzman, A. Hoffman, G. Comtet, L. Hellner, and G. Dujardin, J. Appl. Phys. 86, 4192 (1999); A. Laikhtman, I. Gouzman, and A. Hoffman, Diamond Relat. Mater. 9, 1026 (2000).

${ }^{21}$ F. Himpsel, J. van der Veen, and D. Eastman, Phys. Rev. B 22, 1967 (1980).

${ }^{22}$ M. Kawaguchi, T. Kawashima, and T. Nakajima, Chem. Mater. 8, 1197 (1996); J. Morar, F. Himpsel, G. Hollinger, J. Jordan, G. Hughes, and F. McFeely, Phys. Rev. B 33, 1340 (1986).

${ }^{23}$ P. E. Batson, Phys. Rev. Lett. 70, 1822 (1993).

${ }^{24}$ J.-Y. Raty, G. Galli, C. Bostedt, T. van Buuren, and L. Terminello, Phys, Rev. Lett. 90, 037401 (2003). 